This item was submitted to Loughborough's Research Repository by the author.

Items in Figshare are protected by copyright, with all rights reserved, unless otherwise indicated.

\title{
A case study of the Neti Pot's rise, americanization, and rupture as integrative medicine in U.S. media discourse
}

PLEASE CITE THE PUBLISHED VERSION

http://dx.doi.org/10.1080/10410236.2015.1047145

\section{PUBLISHER}

(C) Taylor \& Francis

\section{VERSION}

AM (Accepted Manuscript)

\section{PUBLISHER STATEMENT}

This work is made available according to the conditions of the Creative Commons Attribution-NonCommercialNoDerivatives 4.0 International (CC BY-NC-ND 4.0) licence. Full details of this licence are available at: https://creativecommons.org/licenses/by-nc-nd/4.0/

\section{LICENCE}

CC BY-NC-ND 4.0

\section{REPOSITORY RECORD}

Ho, Evelyn Y., Kathryn A. Cady, and Jessica Robles. 2019. "A Case Study of the Neti Pot's Rise, Americanization, and Rupture as Integrative Medicine in U.S. Media Discourse”. figshare. https://hdl.handle.net/2134/20702. 
Running head: NETI POTS IN US MEDIA DISCOURSE

A Case Study of the Neti Pot's Rise, Americanization, and Rupture as Integrative Medicine in US Media Discourse

\author{
Evelyn Y. Ho \\ University of San Francisco \\ Kathryn A. Cady \\ Northern Illinois University \\ Jessica S. Robles \\ University of Washington \\ Loughborough University
}




\begin{abstract}
In a period of only one decade in the US, the neti pot shifted from obscure Ayurvedic health device to mainstream complementary and integrative medicine (CIM), touted by celebrities and sold widely in drug stores. We examine the neti pot as a case study for understanding how a foreign health practice became mainstreamed, and what that process reveals about more general discourses of health in the US. Using discourse analysis of US popular press and new media news (1999-2012) about the neti pot, we trace the development of discourses from neti's first introduction in mainstream news, through the hype following Dr. Oz's presentation on Oprah, to 2011 when two adults tragically died after using Naegleria fowleri amoeba-infested tap water in their neti pots. Neti pot discourses are an important site for communicative analysis because of the pot's complexity as an intercultural artifact: neti pots and their use are enfolded into the biomedical practice of nasal irrigation and simultaneously Orientalized as exotic/magical and suspect/dangerous. This dual positioning as normal and exotic creates inequitable access for using the neti pot as a resource for increasing cultural health capital ( $\mathrm{CHC}$ ). This article contributes to work that critically theorizes the transnationalism of CIM, as the neti pot became successfully Americanized. These results have implications for understanding global health practices' incorporation or co-optation in new contexts, and the important role that popularly-mediated health communication can play in framing what healthcare products and practices mean for consumers.

Keywords: Ayurveda, integrative medicine, alternative medicine, cultural health capital, discourse analysis
\end{abstract}


The United States has seen a burgeoning interest in traditional Asian healing practices. From reporter James Reston's introduction of acupuncture in 1971 (Barnes, 1998), ${ }^{1}$ to yoga classes being offered in gyms across the country, it is not surprising that Asian health practices affect and are transformed by mainstream US discourses of health. A recent US health phenomenon, the neti pot, is both a transnational artifact and a linguistic sign that encompasses a uniquely Americanized health practice. The neti pot is a small ceramic, glass, or plastic container with a handle and spout used to pour a warm water saline solution in one nostril, through the nasal cavity, and out the other nostril. It was originally used as part of Ayurveda, a 3000-year-old Indian traditional health system based on knowledge of a person's constitution and life forces (NCCAM, 2013, August) that became standardized in the early $20^{\text {th }}$ century (Reddy, 2002). Ayurvedic treatments include herbal medicine, diets, exercise, yoga, and lifestyle changes, all of which are still commonly used today in India as a complementary medicine alongside biomedicine (NCCAM, 2013, August). Like yoga, which is also used in isolation from Ayurveda, ${ }^{2}$ the neti pot emerged in the last few decades during a time in which biomedicine, especially managed care, was being critiqued for increased iatrogenic diseases, impersonal and over-technologized care, and rising costs (Goldstein, 1999; Reddy, 2002). As an everyday, non-pharmaceutical device that costs as little as $\$ 10$, the neti pot can be easily ignored as a health fad or treatment for minor illnesses. Although the neti pot cannot cure cancer, the ubiquitous and often chronic nature of sinus problems affect as many as 29.6 million (or $12.8 \%$ of) American adults who have sinusitis, or inflammation of the sinuses (Schiller, Lucas, \& Peregoy, 2012). Furthermore, as with the common cold, it is not the severity that claims our attention but rather the everyday nature of the neti pot that makes it worth investigating. The process of normalizing the neti pot in mediated texts reveals important moments of critique and dissent about access to healthcare options, health decision-making, and patient control. The practice of nasal irrigation (rinsing nostrils using any device) had already been biomedically prescribed for chronic sinus conditions (Harvey, Hannan, Badia, \& Scadding, 2007) before neti's recent 
popularity. The neti pot has become one of the most widely-used, natural, and medically effective methods for treating sinus problems (Achilles \& Mdsges, 2013). However, given its Ayurvedic origins and simplistic, non-pharmaceutical application, it can be difficult to classify: is it complementary or biomedical? In this article, we consider the neti pot to be integrative medicine because of its alternative/traditional origins, fit within a natural/holistic medicine movement, and prescription by biomedical providers and institutions.

Public discourse surrounding the neti pot soared after Dr. Mehmet Oz presented the device in April 2007 on the Oprah show. Exhibiting the so-called "Oprah effect," Yahoo! searches for "neti pot" rose $12,325 \%$ the same day, and subsequent sales of neti pots and other nasal saline irrigation devices skyrocketed (Yahoo!, 2011, May 19). In this case study, we trace the development of discourses surrounding the neti pot from its introduction in mainstream news, through the "Oprah effect," to the 2011 tragedies when two people died after using Naegleria fowleri amoeba-infested water in their neti pots. We examine US popular press and new media news (1999-2012) about the neti pot to understand how this foreign health practice became an accepted complementary and integrative medicine (CIM) ${ }^{3}$ treatment. In doing so, we also consider the implications of that process for broader health discourses and health inequalities. Neti pot discourses are an important site for communicative analysis because of the pot's complexity as an intercultural artifact: as both a product (the neti pot) and a practice (neti potting), neti pots are enfolded into the biomedical practice of nasal irrigation and simultaneously Orientalized as exotic/magical and suspect/dangerous. This dual positioning as normal and exotic creates inequitable access for using the neti pot as a resource for increasing cultural health capital (CHC) (Shim, 2010). In the next two sections, we review research about Ayurveda and yoga in the US, as those are the "parent" practices from which neti emerged in India, and then present the health communication literature about $\mathrm{CHC}$ as a way to understand the health context of CIM. 
In order to understand neti's origin, we begin by reviewing research about Ayurveda and yoga the health/lifestyle practices from which the neti pot emerged. Although there is extensive research regarding the medical benefits of yoga (McCall, 2014), fewer studies examine the cultural aspects of yoga or Ayurveda as practiced in the US. The research that does exist recognizes the transnational nature of yoga and Ayurveda in relation to post-colonial India (Langford, 2002), the New Age movement (Reddy, 2002), and appropriation and Americanization of Eastern beliefs (Barnes, 1998). Although for much of its history yoga was a religious practice in India, some have written about yoga's relatively recent transformation in India from religious practice to a symbol advancing Indian nationalism (van der Veer, 2007). In the late $19^{\text {th }}$ century, Swami Vivekananda travelled the world, introducing Hinduism and yoga as a lifestyle practice linking mind and body through exercise (van der Veer, 2007). At that time, the US was particularly receptive having also home-grown various alternative healing forms with spiritual/pseudo-religious underpinnings (such as Christian Science) (Whorton, 2002). Scholars argue that modern yoga, therefore, is as much a transnational creation as it is an ancient Indian tradition (Hoyez, 2007). The form of yoga that gained popularity in the West was one that promised its practitioners a path to spiritual well-being that was explicitly not religious (Hoyez, 2007). Instead, yoga as Indian spirituality - was promoted through pop culture icons like the Beatles, and marketed as a lifestyle or simply exercise (van der Veer, 2007). Similarly, in writing about Ayurveda, Reddy (2004) claims that it is America's love of holism and desire for the metaphysical that gives authority to Ayurveda, not its Indian origins. In the US, the neti pot sits amidst this context of commodified and transnational Asian health care.

\section{The Cultural Health Capital of Complementary and Integrative Therapies}

Cultural health capital is defined as "the particular repertoire of cultural skills, verbal and nonverbal competencies, and interactional styles that can influence healthcare interactions at a given historical moment" (Shim, 2010, p. 2). CHC research recognizes that a provider's response to the 
deployment of a patient's repertoire of cognitive, attitudinal, and behavioral resources can affect interactions and explain how health disparities manifest and are reinforced (Shim, 2010). In this article, we expand the usage of this concept to examine the relationship between $\mathrm{CHC}$, mediated health discourses, and access to health treatments. CHC is an extension of Bourdieu's (1979) concept of cultural capital, which refers to the non-economic practices by which people acquire social status or social mobility (including, for example, ways of dressing and speaking). In the healthcare context, $\mathrm{CHC}$ includes a variety of attitudes and linguistic strategies such as knowledge of medical topics, a pro-active stance toward health, and belief in and resources to practice self-discipline and self-surveillance (Shim, 2010). Mass media play an important role in affecting what particular practices, beliefs, and knowledge lead to greater $\mathrm{CHC}$ given the current health context.

CHC, like cultural capital, is both instrumental and symbolic (Bourdieu, 1979; Shim, 2010). In other words, if we examine the use of neti pot as a resource for increasing $\mathrm{CHC}$, one form of instrumental value is in the benefit to one's sinuses, while symbolic value may be found in the associated cachet one gets with using Oprah/Dr. Oz' hot-new product. $\mathrm{CHC}$ is "developed through enacting cultural practices and appropriating and consuming cultural goods" (Shim, 2010, p. 3). The repeated embodiment of these practices eventually develops a habitus (Bourdieu, 1979), or toolkit of resources and habits, for how to behave in other health situations. Neti users can be understood as developing a particular habitus that associates themselves with other CIM users and movements.

Since the early 1990s, health researchers have paid greater attention to the use of complementary, alternative, holistic, and integrative therapies in the US (Eisenberg et al., 1993). While early research focused on who used these treatments and why, as the sub-field matured, more complex and communication-specific questions have emerged concerning how to communicate integrative medicine (Sharf, Geist Martin, Cosgriff-Hernández, \& Moore, 2012; Sharf, Geist-Martin, \& Moore, 2013) or characterize provider-patient talk about CIM (Koenig, Ho, Yadegar, \& Tarn, 2012), focusing mainly on 
provider-based therapies. Understudied within CIM research are the use of and discussion about nonprovider-based self-care therapies, even though people regularly use CIM self-care to manage chronic illness symptoms (Arcury et al., 2012). In fact, nearly two-thirds of the $\$ 33.9$ billion of out-of-pocket expenses spent on CIM in 2007 were for self-care therapies, amounting to $11.2 \%$ of total out-of-pocket healthcare spending (Nahin, Barnes, Stussman, \& Bloom, 2009).

CIM research illustrates the US health context into which the neti pot was introduced. CIM has been defined by its emphasis on self-care, holistic view of the individual, focus on nature, and wellness promotion (Kaptchuk \& Eisenberg, 1998). People who use CIM weigh costs and potential side effects by using self-knowledge and observation (Kaptchuk \& Eisenberg, 1998; Thorne, Paterson, Russell, \& Schultz, 2002). The early draw of holistic medicine (1960s-80s) as an alternative to biomedicine was based on the sacredness and spirituality of daily health rituals to heal in an overly-technologized world (Kaptchuk \& Eisenberg, 1998). Celebrity health providers like Deepak Chopra and Andrew Weil were instrumental in teaching Americans new health information, legitimizing alternatives, and establishing the mainstream business of CIM (Goldstein, 2002). While the 1990s were a decade in which alternative medicines moved from the economic sidelines of specialty healthcare to a more mainstream corporate path (Goldstein, 2002), the 2000s moved CIM from medicine-at-the-margins toward mainstream acceptance and increased interfacing with biomedicine (Sharf et al., 2012). Integrative medicine can occur in a variety of ways. One way that integration occurs on an individual level is when patients mix various therapies on their own (Boon, Verhoef, O'Hara, Findlay, \& Majid, 2004). On a more complex level, attempts to create institutional spaces for integrative medicine - for example, in clinics with biomedical and holistic health providers - often require "trailblazing" efforts because biomedicine and holistic medicine principles may not always align (Sharf et al., 2012). In these instances, rather than focus on what is biomedical and what is holistic, some authors have argued that CIM is a new form of healthcare altogether (Sharf et al., 2013). 
Some scholars caution against treating biomedicine as the standard while assuming other therapies are merely sanctioned supplements (Baer, 2004). Under this model, the gold-standard of biomedicine - the randomized controlled clinical trial - is used to prove the efficacy and safety of CIM. Although this is one way of integrating therapies, scholars question whether effectiveness, or demonstrated benefit in real-life conditions, as opposed to blinded, controlled conditions, should suffice for demonstrating the value of CIM (Shannon, Weil, \& Kaplan, 2011). In the case of the neti pot, safety and efficacy are not in question. Nasal irrigation (with or without the neti pot) is well-established as efficacious treatment for chronic sinusitis with few side effects (Harvey et al., 2007). However, studies also show that nasal irrigation may not be effective for acute colds or flus, and there is not enough evidence to recommend its use in these cases (Achilles \& MVdsges, 2013; Kassel, 2010). Despite biomedicine's endorsement of nasal irrigation for sinusitis but not colds or flus, if users find the neti pot effective for treating acute conditions, then the gold standard may not be the most important criterion.

Set within this context of CIM and nasal irrigation, the neti pot becomes an important practice and commodity for understanding and expanding the scope of $\mathrm{CHC}$. Cost is a real factor in people's decisions to use CIM (Shannon et al., 2011). As Bishop et al. (2008) write, while some people use CIM as treatment for a particular illness condition, another way people use and understand CIM is as a treat or personal luxury, seeing the actual health need for CIM as less important. Examples include aromatherapy or massage, which, when attached to upscale spas may function as a treat - and a demonstration of one's CHC. This stands in contrast to CIM used as treatment, especially if it is the treatment of choice due to cost considerations. Given how relatively inexpensive the neti pot is as a form of self-care CIM, and its often "gross"-descriptions, it is worth asking where the neti pot fits, as treat, treatment, or both, and whether different levels of $\mathrm{CHC}$ can be attached to its various uses.

The neti pot, with its Ayurvedic origins, is a foreign-originated, self-care therapy and object for delivering what has become standard biomedical treatment. Despite the proliferation of CIM usage, 
there is a surprisingly small amount of research in health communication regarding the normalizing process of Asian health practices in the US (see Ho, 2006). One path toward understanding this process is through examining popular news media discourses. Therefore, in this case study of the neti pot, we ask the following questions:

RQ1: How do mediated texts' depictions of the neti pot change over time?

RQ2: What do discourses of the neti pot say about healthcare more broadly?

RQ3: How does the neti pot function as a form of cultural health capital?

\section{Discourse Analysis and Mediated News Texts}

Our analysis focuses on the different ways discourse about the neti pot, as circulated in a set of mediated texts, constructs the meaning of the neti pot over time. We use discourse analysis (Gee, 2014) to analyze how elements of language frame this social object and practice in texts, linking these specifics to discourses - broader ways of thinking and speaking (Cameron, 2001) - that define and give life or "form" (Foucault, 1972) to objects and ideas in society. This case study analysis reveals some ways in which US health discourses are concretized in texts and become materials for consumers' sense-making. This section overviews this approach to discourse, the data, and how data were analyzed.

Our approach is both descriptive regarding content and structure of discourse, as well as critical regarding how discourse is political insofar as it reflects particular social goods at stake and constructs how those goods should be distributed (Gee, 2014). The social goods under investigation here are health and healthcare access, and $\mathrm{CHC}$ comprises one resource that skilled consumers can use to navigate their health participation. With people increasingly turning to the Internet and other online/mediated sources before or instead of visiting a health provider (Sundar, Rice, Kim, \& Sciamanna, 2011), media can wield considerable power in constructing what health products and practices are deemed most desirable (Hess, 2013). Mediated texts play a role in constructing the meaning of healthcare for potential consumers, thereby becoming a resource for participants from which to make sense and evaluate their 
healthcare options. In choosing the neti pot as a case study, we explicate some ways in which culture and consumption intersect in mediated discourses about health.

This inquiry serves not just to understand the life of the neti pot in the US, but may also shed light on other cases where the meaning of newly-mainstreamed health objects and practices evolves in discourse. Meaning does not arise naturally but is produced in relationships among the social and institutional formations that are most powerful or influential in a society, such as media institutions (Hall, 1996, 1997). The popularization of meaning is the result of ideological processes in which "the languages, the concepts, categories, imagery of thought, and the systems of representation" are deployed by social groups to "make sense of, define, figure out and render intelligible the way society works" (Hall, 1996, p. 26). These ideologies, which define how people see and understand the world, are accomplished through social practices: signs, symbols, language, and so forth used in situations that reproduce dominant or popular meanings (Hall, 1997).

Discourse is an important site at which ideology manifests, in terms of both its productivity and visibility. Discourses have implications for how people should be and act (Gee, 2014). This paper focuses especially on places where texts link larger discourses (macro-discourse or "big D" discourse) to specific communication acts (micro-discourse or "little d" discourse) (Gee, 2014). By examining how thematic ideas about the neti pot are constructed in the texts, we seek to shed light on micro/discursive details of text practices and to show how these implicate macro/Discursive societal issues around health and culture. Our analysis attends to how these discourses get referenced and articulated in specific cases by examining such details as how the neti pot and its practices are referenced; how its meanings are constructed through sentence construction, terminology, and metaphor; and how these textual elements are intertextually reinforced (as when texts copy or quote from others, quote the same sources, or use similar terms) (Gee, 2014).

Data are US popular press and new media news published between 1999 and 2012. Texts were 
gathered from Lexis Nexis Academic using the search term "neti pot*" and included traditional newspapers and magazines, news websites, newswire feeds, press releases and PR wire services, transcripts of broadcasts such as talk shows, blogs, Twitter feeds, trade publications, legal documents, and news aggregators purporting to report news-related items (see Table 1). We removed non-US sources and repeats. For simplicity, we refer to the 277 separate stories as "news," "media," or "texts," highlighting specifics (including media type) as appropriate in the analysis. We examined all types of texts because they represent a broad range of media voices including expert and lay, news and entertainment, personal stories/anecdotes, and lists of links with brief content-related titles.

We began data analysis by open coding every $10^{\text {th }}$ article in chronological order. We developed key terms and ideas and grouped articles together, based on their overarching purposes. If there were coding questions, all authors examined the article(s) in question and reached consensus. This was useful to see broad changes over time. Thereafter we read through the data iteratively to finalize themes and their relationships. Each author shared exemplary quotations. Looking across texts provided insight into how neti pots were discussed and how texts referenced and copied each other - demonstrating variation as well as density in how the discourse configured across this particular slice of media. We also examined the putative onset of the explosion of neti pot discourse - Dr. Oz's appearance on Oprah - as well as its follow-up on the show and the Oprah website.

\section{Case Study of the Neti Pot: From Unknown Exotic to Commercialized Menace}

The first article referencing neti was published on November 16, 1999 in The Detroit News. Fewer than 10 articles appeared each year until 2007 when Dr. Oz introduced the neti pot, after which the number more than tripled compared to the two years prior (see Table 2). A sharp increase in the number of stories is also apparent in late 2011 and 2012, in the wake of the two Naegleria fowleri deaths. The three chronologically-ordered sections of this case study analyze the strategies by which the neti pot was endorsed as a viable health option within existing institutional structures, and how media 
responded to the threat posed by the deaths.

\section{9-April 2007}

This section explores linguistic constructions of the neti pot and its use prior to, and within, Dr. Oz's segment on Oprah in April, 2007. The amount of news coverage in this period is small: 36 articles, or just under $13 \%$ of the total corpus. Although modest in number, these initial articles are important because many ways of understanding the neti pot that are established then remain consistent over time. Even for those frames that change, tracking these changes from the beginning remains key to our analysis. The themes, arguments, and language that established neti pots included their biomedical presentation, their exotic and odd qualities, their link to holistic medicine or yoga, and the access they give patients to natural wellbeing.

Much of what was published from 1999-2005 centered on the development and sale of SinuCleanse neti-related products. Press releases from the company, and local Madison, WI newspaper coverage of the business, established neti pots as biomedically efficacious. , However, this biomedical presentation also relied on the long history of Eastern neti pot use. As mentioned previously, this combined representation fits the form of integrative medicine, in which biomedicine subsumes alternative medicines through scientific testing. For example, a SinuCleanse press release distributed on Business Wire assured those with allergies and sinusitis that "there is hope for instant relief, thanks to an ancient practice that has recently been confirmed by science: nasal wash" ("Doctors, research," 2003, December 8). The neti pot's "ancient" status is a selling point, but most important was that its efficacy had been "confirmed by science." The company also frequently mentioned neti's link to yoga, while simultaneously positioning their product as modern, scientific, and mainstream. For example, The Capital Times newspaper explained, SinuCleanse "is based on the neti pot, which has been used in the practice of yoga for centuries" ("United flight attendants," 2004, December 31), and press releases called SinuCleanse a "mainstream version of the neti-pot" ("Survey shows," 2004, November 30) that 
was "suitable for family home use" ("New study," 2005, September 6). Although much of this early biomedically-framed discourse came from SinuCleanse, it is nevertheless instructive about US news regarding neti more generally. First, throughout the corpus, it remained important that biomedicine found neti efficacious. Additionally, it was unique to these early data that neti pots were consistently linked to yoga specifically. Neti's origin, when mentioned in later data, was typically briefly tagged as Indian or generically ancient.

Other early discourse of the neti pot embodied "the simultaneous allure and menace of the exoticized Eastern other" (Durham, 2001, p. 206). Different strains of American Orientalism (Lowe, 1991) regarding this type of integrative medicine emerged in these early data and continued even after the 2011 deaths. Framing the neti pot as an Oriental alternative/complement to more accepted Occidental sinus treatments (drugs, allergy shots) relied on its oddity. For example, an article listing alternative treatments for allergies (including neti, homeopathy, and acupuncture) described the practices as "The unproven, the strange, the neti pot" (Davenport, 2000, March 21). Also, nearly every article throughout the data explaining how a neti pot is used began by assuming the audience would find the practice "gross," "yucky," or "strange," as in: "If you do it right . . ., the water will run in one nostril, circulate around the ethmoid and sphenoid sinuses between your eyes, and pour out the other nostril. Yuck, I know" (Merrill, 2003, October 14). These sorts of linguistic markers and disclaimers "warned" the reader that the neti pot process was atypical. Such disclaimers usually serve to head off potential criticisms or ill-effects, an important aspect to acknowledge when attempting to sell something new. However, rather than downplaying the negative, many texts highlighted it, using informal language (such as "gross") or colorful metaphors (such as "drowning"), as well as joking about it (for example, that you'd want to hide in the closet to do it). By using disclaimers and qualifiers, these discursive elements construct the neti pot as disgusting/gross/strange in three interrelated ways.

First, these descriptions reference the physical, embodied act of using the neti pot. "Gross" and 
"yucky" are forms of "response cries" (Goffman, 1981), displayed as authentic, almost reflexive responses to something that is seen as grotesque and bizarre. The neti pot is, after all, part of a physical process involving one's nose and its associated fluids. While highlighting the grotesque may be a strange move, it is still within the realm of "good taste": although mucous may be gross, it is not as taboo as other bodily fluids. Furthermore, the grossness is always paired with an emphasis on how effective the practice is; in a way, pointing out its drawbacks may serve to demonstrate how authentic the neti pot and its use is, positioning its lack of glamour as part of what makes it a genuine treatment. Especially in blog posts, but also in print op-ed, presenting one's reaction to the neti pot as that of a "regular" (White, middle class) person further demonstrates that the neti pot and neti potting are bizarre, but work - and you can trust the authors' words precisely because they have endured its oddity.

Second, these apparently-negative descriptions of the neti pot and its practices also construct the neti pot as outside of everyday experience. It is not just gross in the sense of being physically unpleasant to experience or witness, it is also just not what ordinary people do. While these meanings are not unrelated, this one is particularly relevant in the context of commercial media highlighting (a) what is special about the neti pot (as an attention-getting tactic), and (b) what needs to be addressed in order to sell the neti pot. Pointing out the neti pot's oddness is only a selling point insofar as causing initial sales, while reincorporating it as ordinary (as in later texts which compare it to brushing one's teeth) is needed to sell it more widely and keep sales consistent or increasing.

Third, the neti pot's strangeness was attached to its generically foreign and exotic origins. Not only were neti pots subject to a flat construction as a strange and slightly gross oddity that was superficially contextualized as "ancient," but they were also essentialized by the dominant metaphor used to describe the pot: Aladdin's lamp. Twenty-two percent of articles from this time used the Aladdin's lamp metaphor. Although some articles used the equally-descriptive metaphor of a teapot (17\%), the repetition of Aladdin's or genie's lamp to describe an ancient Indian practice can be read as 
Orientalizing (Said, 1979). By conflating an unknown ancient India with a more familiar, although equally exotic, device from a Middle Eastern folk story, these articles presented the neti pot as simultaneously magical, foreign, and preferable over other treatments. For example, the Pittsburgh Post-Gazette highlights the magical nature of Aladdin's lamp, linking it to the neti pot's effectiveness: "Three February wishes: Clear nostrils. Easy breathing. No medicine. For many, relief lies in the neti pot, the Aladdin's lamp of cold prevention" (Gurnett, 2003, February 18). Relying on a dichotomized East/West, in which any part of the East can be conflated with any other part, texts essentializing the neti pot offer similar Orientalist perspectives as attached to Ayurveda (Reddy, 2002) or Chinese medicine (Kaptchuk, 2000) in the US. In other words, unlike medicine, which works scientifically, the preferred explanation for such a simple (and simplistic) natural treatment that works without medicine is that it must be magical.

Having established the Orientalizing combination of ancient, magic, foreign, and simplistic, subsequent stories about neti's natural/drug-free quality reinforced its allure as a biomedical alternative. A typical story stated, "With up to 40 million Americans fighting sinusitus [sic] and tiring of antibiotics, antihistamines and decongestants, [an interviewee] sees water irrigation as a natural alternative" (Gurnett, 2003, February 18). Sales pitches frequently used neti's association with "natural." For example, every SinuCleanse press release at the time linked "natural" and "drug-free" to its product, and these connections continued throughout the corpus regardless of the time period or genre of the text. This alternative character also indexes the natural/holistic movement that emerged in the US in the 1960s-70s, critiquing biomedicine in favor of exotic non-standard treatments (Alster, 1989).

The allure of fetishized Asian holistic/natural medicine was a common frame in early neti news that continued relatively unchanged through the whole corpus, even after the deaths. Before $\mathrm{Dr} . \mathrm{Oz}$, the celebrity doctor associated with neti pot use, was "holistic health guru" Dr. Andrew Weil (Gurnett, 2003, February 18), a public figure with a long, well-respected history in holistic and integrative medicine and specialty audience associated specifically with CIM (Baer, 2003). Published lists of celebrity users would 
usually include reference to the Los Angeles Lakers basketball team. Although never explicitly stated, contemporaneous readers would also likely draw intertextually on associations with NBA coach Phil Jackson (known as the "Zen Master") and Eastern medicine/spirituality. Before Oprah rendered neti pots mainstream, they were identified with celebrities who were publicly linked to holistic or Eastern medicine and who themselves carried particular forms of cultural health capital. Phil Jackson and Dr. Andrew Weil were easily recognizable as leaders in New Age holistic health in the US. As Baer (2003) stated, New Age holistic health is affiliated with two types of clients: those with high disposable incomes (who can afford expensive health retreat centers) or those with alternative lifestyles (who consume mass-produced media, products, and other less expensive holistic treatments). Like other New Age holistic health, neti pot's affordability makes it an accessible way of using CIM, while its affiliation with star-power aligns its users with the more prestigious and luxurious branches of holistic health.

Dr. Oz's presentation of the neti pot on Oprah drew on some of these discursive constructions already in circulation, but framed neti in a more Western biomedical way than did early news discourse. On the April 26, 2007 "Your Questions Answered!" (Oz, 2006, January 1b) segment, Dr. Oz answered a series of health and hygiene questions posed by audience members. A sinusitis sufferer, Amy from Texas, came from the audience to demonstrate using a neti pot. One month later, Amy returned $(\mathrm{Oz}$, 2006, January 1a), and Rachel, an employee working in the show's control booth, was also interviewed. Both reported how much relief they found from regular neti use (Wamacquire, 2010, October 5). The instrumental benefits of the neti, however, are not the only benefits to users. By using the most recent Oz/Oprah approved devices, these very public neti users also gain a particular kind of $\mathrm{CHC}$ affiliated with the mainstream capital of Oz/Oprah.

From the moment Oz introduced neti, or as he called it, "a nose bidet," to Oprah, she played up how "gross" it was with incredulous looks and other physical comedy, gesturing to her nose and questioning how it worked. Although initially describing the pot as a genie lamp, both segments focused 
little on Orientalist views of the neti pot and more on its assumed gross or messy exoticism.

Furthermore, $\mathrm{Oz}$ did not frame neti as $\mathrm{CIM}$, alternative, or specifically Asian. His uncommon label for the device, nose bidet, associated neti with fancy Western hygiene through its reference to the (typicallyWestern European) toilet plumbing fixture used to wash one's nether regions. Framing neti as a Western, high-cultured, hygienic practice dissociated it from the questions of safety so often leveled against alternative medicines, and, instead, attached a more luxurious level of $\mathrm{CHC}$ to the relatively inexpensive product. As the Oprah follow-up segment demonstrated, Oz's Western biomedical view of the neti pot focused on its efficacy as a new form of hygiene that people should learn to use, with no discussion of its safety. Even his presentation of preparation focused on proper use for comfort (water should be "warm" "with some salt in it" to avoid "a head rush"), rather than safety.

\section{April 2007-2011: Neti is the New Normal}

Initial stories in this time period delivered news of the neti pot as a hot new fad. Over time, reports changed tone as neti became more integrated as a regular hygiene and self-care practice for proactive, health-conscious Americans. The ultimate sign that the neti pot had established itself as normal were stories in which neti was used as a metaphor for explaining other unknown health practices. During this time, President Obama also signed the Affordable Care Act, and so we end this section discussing the neti pot's formulation as an alternative form of health insurance.

Neti Hype. Immediately following the Dr. Oz neti segment, neti pot sales increased alongside news coverage about both the pot and its sales (see Table 2). In fact, in the eight months following Dr. $\mathrm{Oz}, 10$ articles were published. Previously, the most published in a year were eight (in 2003). Described as a consumer-driven desirable, stories framed the Oprah episode as the facilitator of this demand and a real turning-point for the neti pot. For example, one store owner is quoted as saying: "Usually, when we sell neti pots, I can go through maybe half a dozen in a week. .. Now, we go through that in a day or less" (Lalley, 2007, July 17, p. D1). Likewise, a neti pot manufacturer stated, "'The phone started ringing 
off the hook' as soon as the segment was finished" (Ward, 2008, February 17). While the news articles acknowledged Dr. Oz and Oprah as the source of hype, it is also worth mentioning that the articles themselves functioned both explicitly and implicitly as sales devices. The articles promoted new products, quoted prices, and listed places where products could be purchased. It is well documented that we live in an historical moment in which advertising permeates US news (Anderson, 1995; McChesney, 1999) and as mentioned, celebrity health experts often mainstreamed CIM therapies for mass audiences (Goldstein, 1999). News covering Oz/Oprah and neti fit these trends.

The neti pot's association with both ancient origins and current celebrity lifestyle was pervasive throughout these texts, and functioned to further Americanize the practice of neti potting. One article claimed "this ancient Hindu practice of schnoz-cleansing has become a super-hot trend" (Poist, 2007, July 1). Besides Oprah and Dr. Oz, other celebrities were also quoted, such as Kathie Lee Gifford, who proclaimed on the Today Show: "I love the neti pot. . . . it's nasty and so much fun" (Nash, 2012, December 18). All of these presentations represent an East-West flow of culture and style (Shome, 2006) that is not neutral. As Durham (2001) notes, "the cultural appropriation of Eastern cultures as trends ... can be understood in terms of issues of imperialism and dominance" (p. 205). Although neti is an Eastern practice migrating West, because "Western countries control the means of media production and marketing" (Durham, 2001, p. 205), Western presentations of the neti pot as a commodity and celebrity fad also function to transform it into an Americanized health practice devoid of its origins and other possible meanings. The irreverence in celebrity news about neti brings into sharp relief the colonial power structure to which this Indian device still falls prey via its entrance into US media.

Integrated Neti: New Forms of Hygiene, New Scope of Healing. After Dr. Oz's introduction of the nose bidet, neti potting was gradually compared to other hygiene rituals including "washing hands, exercising, eating nutritious foods, reducing stress and brushing your teeth" ("Health educator," 2009, October 9). Establishing a new hygiene ritual for the health-conscious laid the groundwork for new neti 
products and further integrated the neti pot into mainstream healthcare. Take, for example, the Betti Pot, a trademarked porcelain neti pot named after its creator Isabella "Betti" Jones. It was marketed as “the only $100 \%$ 'green' sinus cleansing system made in the U.S.A. from high-fired porcelain and sustainable, non-toxic and recyclable materials, to help Americans stave off colds, virus, the flu and other sinus ailments" ("Health educator," 2009, October 9). This press release capitalizes on all the elements of holistic health including personal responsibility for health, nutrition, stress-reduction, link to environmentalism, and for good measure (and to move neti beyond its foreign origins), a made-in-theUSA badge reminding users that the Betti Pot is "for Americans." The turn towards the ritual and hygienic aspects of the neti pot promote the device for self-surveillance and personal responsibility, both key to increasing $\mathrm{CHC}$.

The neti pot was also presented as giving users greater control over their healthcare: "Not only can patients reduce the need for antibiotics or surgery ... but it's one of the rare medical treatments where patients are in control" (Shute, 2007, November 29). In this case, control literally means self-care because patients do the neti potting themselves. Control is important in the US health context where patients often feel little power over their health decisions (Charles, Gafni, \& Whelan, 1999) and often use self-care CIM as a way to take responsibility for their chronic conditions (Thorne et al., 2002). The explicit connection between CIM usage and American identity continued as one story in 2012 explicitly claimed that Americans "hate to be passive," and the neti pot allows "us" to "take action" when we are sick (Paster, 2012, February 8). Promoted as a device for healthy Americans to take charge of their health, using the neti pot establishes oneself as a person with $\mathrm{CHC}$.

One signal that the neti pot was well-established and normalized was when stories began to appear in which the neti pot and neti potting were used to explain newer (often stranger) but similar practices. For example, an article about halotherapy explained these salt spas are "like ... sitting in a Neti Pot" (Heckel, 2011, September 20). Uses of neti as a metaphor continued throughout the data set 
even during and after the Naegleria fowleri deaths were reported. For example, the act of cleaning out one's nose with a neti pot was used by a personal finance blogger to explain how getting organized releases stress ("How to work," 2012, February 15). These metaphorical uses were evidence of neti's normalized status in mainstream mediated discourse and its increasing visibility as a resource for CHC.

Neti is Affordable Insurance. Although the neti pot was often presented as CIM, it was also presented as a form of health insurance alternative to more expensive insurance-based biomedicine. One celebrity called the combination of neti and an over-the-counter supplement "my insurance policy" (Brickman, 2012, January 15). This metaphorical use of insurance was joined by other articles discussing neti as an inexpensive alternative because interviewees were actually uninsured. In the recent US climate in which health insurance is a part of everyday conversation, insurance became a buzzword in news coverage surrounding the passage of the Patient Protection and Affordable Care Act on March 23, 2010. An article profiling a young woman with temporary work and no health insurance stated: When she came down with a sinus infection two years ago, she called her mother. ... Her mother, who has chronic sinus problems, passed along her own doctor's advice: use a neti pot. It worked. Brewster said she worries about getting older with no health insurance. How am I gonna take care of myself, medically, if I get sick? (Davis, Salit, \& Arditi, 2010, March 16)

This story is peppered with inexpensive home remedies the woman uses because she cannot afford biomedical care: the neti pot, vitamins, healthy diet, and stress avoidance. The last lines provide an interruption in her list of successful home remedies: despite their effectiveness, they are inadequate solutions for both the more serious illnesses faced by older adults and the broken system of US healthcare, in which not having insurance means not having medical care. Presented as a treatment, not a treat, this and other examples presented the neti pot as a low-cost home remedy used out of necessity - not as a quirky, celebrity-endorsed lifestyle choice for those who already have insurance. In these cases, the neti pot was not a ritual habitus that offers its users distinction. Instead, the use of the neti, as 
a necessity, became a marker of that lack of health care access. While CIM is generally used by the more affluent (Barnes, Bloom, \& Nahin, 2008), the neti pot's affordability associates it with a broader economic audience. Therefore, it is not the neti pot itself that offers its users (or promoters) guaranteed increases in luxury-based CHC. In fact, it is so cheap that some stories spoke about this as an economic liability. For example, in a USNews.com story, one medical school professor is quoted as stating, "There isn't a company that's making a huge amount of money off of salt water" (Shute, 2007, November 29). The article continues that because there is little profit potential, "doctors often forget to mention it," resulting in the general public not knowing about neti as much as other sinus remedies (Shute, 2007, November 29). But companies did find ways to sell the pot and as the next section describes, encouraging consumption became even more important after two deaths.

\section{Rupture in 2011: The Danger of the Neti Pot}

After the neti pot's rise to popularity, much of the discourse seemed to have successfully managed the dilemma between the neti pot's quirky faddishness, and its potential as a viable, everyday form of healthcare. However, this balance was disrupted. A rupture is an interruption in the continuity of history, a moment in which what appeared to be stable becomes uncertain (Foucault, 1972), and the work of maintaining the stability of institutions becomes visible. In August 2011, news emerged indicating that three Southern US residents were killed by an amoeba called Naegleria fowleri, two from swimming and one via using unboiled tap water in a neti pot. By December 2011, a second death had been linked to neti use, and headlines shifted from death-by-amoeba to death-byneti pot. By chronologically examining coverage of the deaths, we uncover what underlying meanings might be (re)activated about the neti pot in a time of pressure and mistrust.

Although few articles concluded that the neti pot was to blame for the deaths, many early stories placed the neti pot in headlines and at the center of news coverage, thus making its culpability relevant. This link continued even after the water was found to be the cause of death. This feature of 
the texts reflected and re-emphasized two earlier ways of talking about the neti pot: (a) collapsing the object (neti pot) with the broader process of nasal irrigation, a metonymy in which the former stood in for the latter; and (b) again emphasizing the strange/foreignness of the neti pot, reversing earlier normalization efforts and positioning neti as potentially dangerous.

Headlines often constructed neti pots themselves as the cause of death. Blog headlines tended toward hyperbole: "Death by Neti Pot" (Harshman, 2011) or "Even one death is too many-WE MUST BAN NETI POTS!" (“January 3 Roundup, " 2012). However, even in traditional news stories that offered more complicated analysis of the deaths, headlines focused on neti as the cause. For example, a commonly repeated quotation from Louisiana public health officials, warned, "If you are irrigating, flushing or rinsing your sinuses, for example, by using a neti pot, use distilled, sterile or previously boiled water .... Tap water is safe for drinking, but not for irrigating your nose" (Blazek, 2011, December). This quotation clearly demonstrates that the deaths are caused by water problems, and perhaps secondarily by nasal irrigation, however, the headline to this story - "Rare amoeba infection kills second neti pot user" - implies a different causation. In this headline, the victims are "neti pot users," not people who irrigated their sinuses with unboiled tap water. Even within articles attempting to report the real cause of the deaths, sentence structure could cause ambiguity. In the case of "The FDA warns against the improper use of neti pots which may have caused two deaths last year" ("Lab notes," 2012, August 25), the word "which" was almost certainly meant to be connected to the entire phrase "the improper use of neti pots." However, one might easily read "which" as connecting only to "neti pots." This established a link between two newsworthy elements for commercial media: weird deaths, and weird foreign objects. After the initial flurry of stories citing the "neti deaths," subsequent articles separated the cause of death from the neti pot as an object. Once it became clear that the water and not the device was the problem, it became incumbent upon municipal water companies to deflect blame. How did microbeinfested water, a local public health issue, make it past health and safety regulations? Multiple stories 
reporting on the contaminated drinking water in Louisiana used the opportunity to make sure local readers knew about the safety of their own water. Some did this by claiming that there were no amoebae in local water while others emphasized the safety of drinking the water. For example, Austin Water in Texas assured people that "Drinking tap water contaminated with the amoeba does not create harm. Consequently, the Austin Water utility does not test for the amoeba" (Roser, 2011, December 30, p. B1). Because Naegleria fowleri is killed by stomach bacteria but can pass through the blood-brain barrier via the nasal passage, it becomes a threat only when inhaled. Without explicitly commenting on the neti pot, these stories nevertheless code the practice as strange by way of contrast with the official expectation that tap water will not be used in a nose.

Discourses of neti-deaths and assumptions of tap water used only for drinking relied on the underlying remnants of neti's foreignness and its associated yellow peril. Asians and Asian Americans have throughout US history been portrayed in media as dangerous to mainstream (White) Americans (Ono \& Pham, 2009). This yellow peril, generally associated with Asian people, can also seen with Asian objects. For example, although Naegleria fowleri typically kills swimmers, no articles about the Louisiana deaths noted swimming as a way people contract Naegleria fowleri and how exceedingly rare it is to contract the microbe from nasal irrigation. While no articles explicitly linked the neti pot foreignness with its danger, the continued description of the neti pot as ancient or Indian reinforced this connection.

Over time, after it became clear that the pot itself was not dangerous, and following assurances from water companies, blame for the Naegleria fowleri deaths shifted to individual's incorrect use of the neti pot, specifically in water preparation. Official sources such as a public health alert stated that the problem was with using water for nasal irrigation that had not been "distilled, boiled or sterilized" (Roser, 2012, January 15, p. D2). Celebrities who had endorsed the neti pot, as well as other health professionals, largely took this route. As Dr. Oz and co-author and fellow celebrity MD Dr. Roizen stated: “We don't intend to give up our neti pots and we don't think you need to either. But like so many 
drugstore purchases - aspirin to ice packs - misuse can cause big trouble" (Roizen \& Oz, 2012, January 9, p. 2C). In this example, the position of the doctors as experts and neti pot users as potential troublemakers presents a strong case for the object being safe, while misuse is the problem. The implied conclusion was that it was neither the neti pot nor the water that was really to blame, but the neti pot users who failed to take necessary precautions. Rather than demonstrating one's CHC and health competence, using the neti pot with tap water became framed as a lack of self-surveillance and misuse after the deaths. Yet, this characterization is unreasonable given that no news detailing neti use contained instructions to use boiled or sterilized water prior to 2011 (nor did many instructions that came with neti pots), indicating that water preparation was not a concern that was at the forefront of experts' minds until after the deaths made it relevant. Even in the original Dr. Oz segment, the recommendation for using warm water was a matter of comfort, not safety.

With responsibility increasingly directed toward individuals using neti pots, companies began marketing products using language about safety. This shift illustrates how the shaky ground under the neti pot could be exploited for a new commodification process. Marketing new products decoupled the neti-pot-equals-nasal-irrigation assumption by offering alternative irrigation devices and practices. Press releases cast the neti pot in a negative light so as to sell ostensibly-safer products: "Because [our product] is a sterile, closed system, risks like those being reported with the Neti Pots should not be an issue and patients will get the benefits of sinus rinsing without worry" ("Reported health risks," 2012, January 12). Unlike the neti pot, new devices were designed to guarantee against user error. Companies played on assumed danger: "With this latest, unfortunate, news where municipal water appears to have been unsafe, the risk in using a neti pot, which is basically a 'home remedy', becomes a bit more apparent" ("Sinox spray," 2011, December 21). In addition to calling attention to the water and the neti pot, the reference to a "home remedy" is telling. This invocation sets up an implied contrast between the more desirable newly-technologized medicine (such as the product being promoted) and the 
ancient, unsafe practices deployed in basic home remedies. However, by later referring to "all the goodness of the neti pot," this press release was also able to affiliate with CIM legitimation while simultaneously presenting a kind of neti pot 2.0, saving the good while improving the bad.

From the "terror" in the neti pot to the "deadly killer" in the kitchen tap (Stebner, 2012, April 24), the deaths in Louisiana posed a problem for the neti pot, which had been so enthusiastically endorsed by celebrities and health officials alike, and consumed so readily by people looking for lowcost, drug-free alternatives for sinus care and the newest hot fad. The neti pot, with its quirky practice and foreign antecedents, seemed a perfect scapegoat for the Naegleria fowleri deaths. Casting out that which is fringe and foreign is easier than dealing with a problem closer to home, and the combination of a freaky "brain-eating microbe" and the "Aladdin's lamp of sinus care" made for good news in terms of oddity and shock value. These discourses challenged the $\mathrm{CHC}$ potential of the neti pot, causing some reconfiguration of the commodification process as new products sought to distance themselves from the deaths and even from the original neti pot. The association between the neti pot and the deaths continued to linger despite the vindication of the neti pot. But overwhelmingly, conclusions were positive: the neti pot is not only safe, it works. Indeed, even during and since the deaths, most neti pot news was not about the deaths. So while the memory of the deaths and their link to the neti pot may cause this continued questioning of the neti pot itself, in the end, the misuse of the neti pot - a critical lack of user-self-discipline - was constructed in news as the ultimate source of risk. This further emphasizes the $\mathrm{CHC}$ potential of the neti pot by making certain kinds of knowledge and commodified products necessary for its proper use and distancing those proper forms of neti from the incorrect and unsafe (i.e., home remedy) forms.

The shift of responsibility onto individual neti pot users should perhaps come as no surprise. After all, four powerful US institutions - entertainment, health, business, and mass media - circulated discourses of neti pot praise and had a stake in saving the neti pot, not demonizing it. As demonstrated 
in the quick shift of blame onto and later away from municipal water companies, media discourse diverted attention from any potential structural or institutional problems, revealing a larger societal process through which the status of powerful institutions is maintained by attributing problems to the errors of individuals - a common practice in individualist health research (Zoller \& Kline, 2008). In the case of neti pot news, individual misuse could then be remedied through a renewed called for selfsurveillance, new technological products, and subsequent marketing. As Fairclough (2003) notes in talking about capitalism, challenges to the system can be absorbed into it (as when an "alternative" healthcare method is officially endorsed by the biomedical establishment) or relegated beyond it (as when serious problems like death can ultimately be attributed to the users and not the system itself). The rupture of unexpected deaths following the soaring popularity of the neti pot confirmed the processes of normalization and commodification that were necessary to enfold the neti pot into existing health discourses, turning what could have been a threat into a profit.

\section{Conclusions}

Our research began with the following questions: 1) How do mediated texts' depictions of the neti pot change over time? 2) What do discourses of the neti pot say about healthcare more broadly? 3) How does the neti pot function as a form of cultural health capital? This case study of mediated texts' depictions of the neti pot examined its transformation over time from relatively unknown exotic alternative, to hot new biomedical Oprah-endorsed fad, to Americanized hygiene practice. The neti pot's final transformation was triggered by a rupture focused on neti pot safety. Using discourse analysis to examine what we termed the "meso-discursive" brings into focus invisible strategies through which micro/discursive details of media texts link to larger societal issues around health and culture. By considering the Americanization of this rather mundane healthcare item, we demonstrate some of the broader ideological processes at work when media, health, consumption, and culture collide in discourse, and what this can reveal about discourses of health. Tracking neti's Americanization 
contributes to work that critically theorizes the transnationalism of CIM by offering insights into the evolving ideological construction of health and consumption in the context of cultural appropriation. Such work explores how processes of globalization and localization develop today, in a context in which "American" media and neoliberal economics have displaced the previous colonial relations between countries as the governing logic of global relations (Hall, 1997). The analysis offered here may also be instructive to understanding other sites of acclimation and struggle between alternative practices and the Western biomedical establishment, both Eastern (e.g., cupping or Chinese herbal medicine) and Western (e.g., chiropractic or homeopathy).

Finally, this case study of the neti pot adds to our understanding of $\mathrm{CHC}$. Whether framed as CIM or biomedical, the neti pot's grand introduction on Oprah and subsequent framing as normal hygiene practice created a new mode and a new set of commodities for consumers to access $\mathrm{CHC}$. Oprah's viewers, who presumably have access to biomedical care and clean water, are treated with the latest cosmopolitan upper-class fashions from around the globe. Although the neti pot may help consumers to be proactive health seekers, the neti pot's popularity may also obscure important critiques of healthcare, the cost of health insurance, and even water safety. The status of neti as a lowcost necessity for some and as a specialized knowledge linked to celebrity culture for others illustrates the persistent social inequalities that inequitable access to healthcare exacerbate. Yet, although the "treat" version of neti use is clearly based on acquiring and increasing CHC, the "treatment" version also produced knowledge and self-efficacy for less wealthy users to cure ailments through a home remedy. This aspect of CIM - when it is used not as choice but as necessity - is one that is understudied and had it not been for the deaths, may have been examined further in news about the Affordable Care Act. Instead, the deaths drew public attention to the safety of neti as CIM and to individual responsibility for that safety. Suddenly, unwitting neti users did not have $\mathrm{CHC}$ as a new water preparation was publicized. Because discourses are as much about what is not said as what is said, it is worth noting that 
few stories reported if or how the neti pot is used by ethnic Indians as part of larger Ayurvedic practices; these are not considered newsworthy in the US. Like yoga and Ayurveda, the neti pot may be a transnational production responding to American desires and needs more so than something authentically Indian. In this milieu, the neti pot's actual origins do not matter. Instead, the neti pot is offered to American consumers hungry for a way to take control of their bodies in the latest natural, holistic, and ritualistic ways. The neti pot, thus, provides some users access to $\mathrm{CHC}$ through a number of resources, including individual agency, biomedical efficacy, natural and holistic ideologies, and celebrity affiliation. However, not all users can claim $\mathrm{CHC}$ equally. For example, those using neti as a treat, those with amoeba-free water, and those able to buy the latest and safest neti devices can consistently advance their own pursuit of health through the neti. For other users, the neti pot is not always guaranteed to advance their healthcare or their social status.

This study is limited in that we examined news discourse that was compiled by Lexis Nexis. Future research should directly investigate how neti pot users engage with this device/practice using ethnographic research, interviews or focus groups, especially in light of the discussion around CHC. Does a patient's expression of neti use affect health interactions? Are neti users perceived as proactive, knowledgeable patients, or as alternative ones? Exploring how users actually engage with the neti pot (as choice/necessity, as a tool for developing $\mathrm{CHC}$, as alternative or $\mathrm{CIM}$ ) would expand understandings of integrative medicine in the US. As more and more ancient Asian health practices are popularly adopted into the US, it is important to analyze exactly what happens in the transformation from ancient Asia to modern Americanized Asian practices. 
References

Achilles, N., \& MVdsges, R. (2013). Nasal saline irrigations for the symptoms of acute and chronic rhinosinusitis. Current Allergy and Asthma Reports, 13(2), 229-235. doi: 10.1007/s11882-0130339-y

Alster, K. B. (1989). The holistic health movement. Tuscaloosa, AL: The University of Alabama Press. Anderson, R. (1995). Consumer culture and TV programming. Boulder, CO: Westview Press.

Arcury, T. A., Grzywacz, J. G., Neiberg, R. H., Lang, W., Nguyen, H., Altizer, K., . . Quandt, S. A. (2012). Older adults' self-management of daily symptoms: Complementary therapies, self-care, and medical care. Journal of Aging Health, 24(4), 569-597. doi: 10.1177/0898264311428168

Baer, H. A. (2003). The work of Andrew Weil and Deepak Chopra--two holistic health/New Age gurus: A critique of the holistic health/New Age movements. Medical Anthropology Quarterly, 17(2), 233250.

Baer, H. A. (2004). Toward an integrative medicine. Walnut Creek, CA: Alta Mira.

Barnes, L. L. (1998). The psychologizing of Chinese healing practices in the United States. Culture, Medicine and Psychiatry, 22, 413-443. doi: 10.1023/A:1005403825213

Barnes, P. M., Bloom, B., \& Nahin, R. L. (2008). Complementary and alternative medicine use among adults and children: United States, 2007: CDC National Health Statistics Report \#12.

Bishop, F. L., Yardley, L., \& Lewith, G. T. (2008). Treat or treatment: A qualitative study analyzing patients' use of complementary and alternative medicine. American Journal of Public Health, 98(9), 1700-1706.

Blazek, N. (2011, December). Rare amoeba infection kills second neti pot user. The Clinical Advisor. Retrieved from http://www.lexisnexis.com

Boon, H., Verhoef, M., O'Hara, D., Findlay, B., \& Majid, N. (2004). Integrative healthcare: Arriving at a working definition. Alternative Therapies in Health and Medicine, 10(5), 48-56. 
Bourdieu, P. (1979). Distinction: A social critique of the judgement of taste (R. Nice, Trans.). Cambridge, MA: Harvard University.

Brickman, S. (2012, January 15). 4-hour guru off the clock; author Tim Ferriss has strong likes-knives and kettelbells-but a weakness for Chardonnay. The San Francisco Chronicle, p. L4. Retrieved from http://www.lexisnexis.com

Cameron, D. (2001). Collecting data: Practical and ethical considerations. Working with spoken discourse. London: Sage.

Charles, C., Gafni, A., \& Whelan, T. (1999). Decision-making in the physician-patient encounter: Revisiting the shared treatment decision-making model. Social Science \& Medicine, 49(5), 651661. doi: http://dx.doi.org/10.1016/S0277-9536(99)00145-8

Davenport, K. (2000, March 21). New season means misery for allergy sufferers. Sante Fe New Mexican, p. A-1. Retrieved from http://www.lexisnexis.com

Davis, P., Salit, R., \& Arditi, L. (2010, March 16). The uninsured profile: Young, middle aged, employed; health care. Providence Journal, p. 9. Retrieved from http://www.lexisnexis.com

Doctors, research say drug-free nasal wash relieves sinus problems. (2003, December 8). Business Wire. Retrieved from http://www.lexisnexis.com

Durham, M. G. (2001). Displaced persons: Symbols of South Asian femininity and the returned gaze in U.S. media culture. Communication Theory, 11(2), 201-217. doi: 10.1111/j.14682885.2001.tb00239.x

Eisenberg, D. M., Kessler, R. C., Foster, C., Norlock, F. E., Calkins, D. R., \& Delbanco, T. L. (1993). Unconventional medicine in the United States--Prevalence, costs, and patterns of use. The New England Journal of Medicine, 328(4), 246-252.

Fairclough, N. (2003). Analyzing discourse: Textual analysis for social research. London, UK: Routledge. 
Foucault, M. (1972). The archaeology of knowledge (A. M. Sheridan Smith, Trans.). New York: Pantheon Books.

Frawley, D. (2005). Neti: Healing secrets of yoga and Ayurveda. Twin Lakes, WI: Lotus Press.

Gee, J. P. (2014). An introduction to discourse analysis: Theory and method (4th ed.). New York: Routledge.

Goffman, E. (1981). Forms of talk. Philadelphia, PA: University of Pennsylvania Press.

Goldstein, M. S. (1999). Alternative health care. Philadelphia, PA: Temple University Press.

Goldstein, M. S. (2002). The emerging socioeconomic and political support for alternative medicine in the United States. The Annals of the American Academy of Political and Social Science, 583(1), 44-63. doi: $10.1177 / 000271620258300104$

Gurnett, K. (2003, February 18). Breathe better in winter; Neti pot spells relief for your dry, stuffy nose. Pittsburgh Post-Gazette, p. F-6. Retrieved from http://www.lexisnexis.com

Hall, S. (1996). The problem of ideology: Marxism without guarantees. In D. Morely \& K.-H. Chen (Eds.), Stuart Hall: Critical dialogues in cultural studies (pp. 24-45). New York: Routledge.

Hall, S. (1997). The local and the global: Globalization and ethnicity. Cultural Politics, 11, 179-187.

Harshman, M. (2011, December 21 ). Death by neti pot [Web log post]. Live Well Blog. Retrieved from http://www.lexisnexis.com

Harvey, R., Hannan, S. A., Badia, L., \& Scadding, G. (2007, Jul 18). Nasal saline irrigations for the symptoms of chronic rhinosinusitis. Cochrane Database of Systematic Reviews (3), CD006394. doi: 10.1002/14651858.CD006394.pub2

Health educator Isabella Jones urges Americans to practice daily sinus cleansing to help prevent colds and the flu;--A natural way to keep your family healthy: The Betti Pot(TM) Sinus Cleansing System. (2009, October 9). PR Newswire. Retrieved from http://www.lexisnexis.com Heckel, A. (2011, September 20). Breathe in. Daily Camera. Retrieved from http://www.lexisnexis.com 
Hess, K. (2013). Tertius tactics: "Mediated social capital" as a resource of power for traditional commercial news media. Communication Theory, 23(2), 112-130. doi: 10.1111/comt.12005

Ho, E. Y. (2006). Behold the power of Qi: The importance of Qi in the discourse of acupuncture. Research on Language and Social Interaction, 39, 411-440. doi: 10.1207/s15327973rlsi3904_3

How to work when you're overwhelmed [Web log post]. (2012, February 15). Christian Personal Finance. Retrieved from http://www.lexisnexis.com

Hoyez, A.-C. (2007). The "world of yoga": The production and reproduction of therapeutic landscapes. Social Science and Medicine, 65, 112-124.

Kaptchuk, T. J. (2000). The web that has no weaver (2nd ed.). New York: Congdon \& Weed.

Kaptchuk, T. J., \& Eisenberg, D. M. (1998). The persuasive appeal of alternative medicine. Annals of Internal Medicine, 129, 1061-1065.

Kassel, J. C. (2010). Saline nasal irrigation for acute upper respiratory tract infections. Cochrane Database of Systematic Reviews (3), CD006821. doi: 10.1002/14651858.CD006821.pub2

Koenig, C. J., Ho, E. Y., Yadegar, V., \& Tarn, D. M. (2012). Negotiating complementary and alternative medicine use in primary care visits with older patients. Patient Education and Counseling, 89(3), 368-373. doi: http://dx.doi.org/10.1016/j.pec.2012.02.020

Lab Notes: Overweight kids more prone to gallstones [Web log post]. (2012, August 25). CalorieLab. Retrieved from http://www.lexisnexis.com

Lalley, H. (2007, July 17). Magic lantern; many neti pot users say they're breathing easier these days, thanks to a small ceramic vessel that's making sinus problems disappear. Spokesman Review, p. D1. Retrieved from http://www.lexisnexis.com

Langford, J. M. (2002 ). Fluent bodies: Ayurvedic remedies for postcolonial imbalance. Durham, NC: Duke. Lowe, L. (1991). Critical terrains: French and British Orientalisms. Ithaca: Cornell University Press. 
McCall, M. C. (2014). In search of yoga: Research trends in a western medical database. International Journal of Yoga, 7, 4-8. doi: 10.4103/0973-6131.123470

McChesney, R. (1999). Rich media, poor democracy. Urbana, IL: University of Illinois Press.

Merrill, M. (2003, October 14). Sinus infection remains a pain in winter. Buffalo News. Retrieved from http://www.lexisnexis.com

Nahin, R. L., Barnes, P. M., Stussman, B. J., \& Bloom, B. (2009). Costs of complementary and alternative medicine (CAM) and frequency of visits to CAM practitioners: United States, 2007. National health statistics reports; no 18. Hyattsville, MD: National Center for Health Statistics.

Nash, D. (Executive Producer). (2012, December 18). Today Show [Television broadcast]. New York: NBC. Retrieved from http://www.lexisnexis.com

National Center for Complementary and Alternative Medicine. (2013, August). Ayurvedic Medicine: An Introduction. (NCCAM Publication No. D287). http://nccam.nih.gov/health/ayurveda/introduction.htm.

New study says "wash your nose," shows natural nasal washing relieves chronic sinus symptoms; lowering SNOT-20 scores and improving quality of life over time, without drugs (2005, September 6). Business Wire. Retrieved from http://www.lexisnexis.com

Ono, K. A., \& Pham, V. N. (2009). Asian Americans and the media. Cambridge, England: Polity.

Oz, M. (2006, January 1a). Dr. Oz answers your questions. Retrieved from http://www.oprah.com/health/Dr-Oz-Answers-Burning-Medical-Questions/12

Oz, M. (2006, January 1b). Your questions answered! Retrieved from http://www.oprah.com/health/Your-Questions-Answered/5

Paster, Z. (2012, February 8). Beware of neti pot parasites. Wisconsin State Journal, p. C3. Retrieved from http://www.lexisnexis.com 
Poist, P. (2007, July 1). Ancient yoga nose pose has many new fans. Sunday News, p. G1. Retrieved from http://www.lexisnexis.com

Reddy, S. (2002). Asian medicine in America: The Ayurvedic case. Annals of the American Academy of Political and Social Science, 583, 97-121. doi: 10.1177/000271620258300107

Reddy, S. (2004). The politics and poetics of "magazine medicine": New Age Ayurveda in the print media. In R. D. Johnston (Ed.), The politics of healing (pp. 207-229). New York: Routledge.

Reported health risks of neti pots do not extend to all sinus rinse products. (2012, January 12). PR Newswire. Retrieved from http://www.lexisnexis.com

Roizen, M., \& Oz, M. (2012, January 9). Misuse likely to blame for neti-pot deaths in Louisiana. Denver Post, p. 2C. Retrieved from http://www.lexisnexis.com

Roser, M. A. (2011, December 30). After death, health officials warn against tap water in neti pots. Austin American-Statesman, p. B1. Retrieved from http://www.lexisnexis.com

Roser, M. A. (2012, January 15). Unplugged: Best of Statesman.com and local interest blogs. Austin American-Statesman, p. D2. Retrieved from http://www.lexisnexis.com

Said, E. W. (1979). Orientalism. New York: Vintage Books.

Schiller, J., Lucas, J., \& Peregoy, J. (2012). Summary health statistics for U.S. adults: National Health Interview Survey, 2011.

Shannon, S., Weil, A., \& Kaplan, B. J. (2011). Medical decision making in integrative medicine: Safety, efficacy, and patient preference. Alternative and Complementary Therapies, 17(2), 84-91.

Sharf, B. F., Geist Martin, P., Cosgriff-Hernández, K.-K., \& Moore, J. (2012). Trailblazing healthcare: Institutionalizing and integrating complementary medicine. Patient Education and Counseling, 89(3), 434-438. doi: http://dx.doi.org/10.1016/i.pec.2012.03.006 
Sharf, B. F., Geist-Martin, P., \& Moore, J. (2013). Communicating healing in a third space: Real and imagined forms of integrative medicine. In L. M. Harter \& Associates (Eds.), Imagining new normals (pp. 125-147). Dubuque, IA: Kendall Hunt.

Shim, J. K. (2010). Cultural health capital: A theoretical approach to understanding health care interactions and the dynamics of unequal treatment. Journal of Health and Social Behavior, 51(1), 1-15. doi: $10.1177 / 0022146509361185$

Shome, R. (2006). Transnational feminism and communication studies. The Communication Review, 9(4), 255-267. doi: 10.1080/10714420600957266

Shute, N. (2007, November 29). Stuffy nose or sinus problems? Here's a fix. U.S.News.com. Retrieved from http://www.lexisnexis.com

Sinox spray offers safe alternative to neti pot health risk. (2011, December 21). PR Newswire. Retrieved from http://www.lexisnexis.com

Stebner, B. (2012, April 24). Now even TAP WATER isn't safe! Brain-eating amoeba in $99 \%$ of cases "could come from your own faucet." MailOnline. Retrieved from http://www.lexisnexis.com

Sundar, S. S., Rice, R. E., Kim, H.-S., \& Sciamanna, C. N. (2011). Online health information: Conceptual challenges and theoretical opportunities. In T. L. Thompson, R. Parrott, \& J. F. Nussbaum (Eds.), The Routledge Handbook of Health Communication (2nd ed., pp. 181-202). New York: Routledge.

Survey shows sinus sufferers fed up with over-the-counter drugs; The "search for sinus relief" finds answers in alternative remedies. (2004, November 30). Business Wire. Retrieved from http://www.lexisnexis.com

Thorne, S., Paterson, B., Russell, C., \& Schultz, A. (2002). Complementary/alternative medicine in chronic illness as informed self-care decision making. International Journal of Nursing Studies, 39(7), 671-683. doi: http://dx.doi.org/10.1016/S0020-7489(02)00005-6 
United flight attendants threaten work stops. (2004, December 31). The Capital Times, p. 8B. Retrieved from http://www.lexisnexis.com

van der Veer, P. (2007). Global breathing: Religious utopias in India and China. Anthropological Theory, 7(3), 315-328. doi: 10.1177/1463499607080193

Wamacquire. (2010, October 5). Oprah talking about SinuCleanse [Video file]. Retrieved from http://www.youtube.com/watch?v=e22Ta7iRgks\&list=PL1dyEMdyv1mape9MdLSa9ncQxwbGjU $\underline{\text { A2s\&index }=2}$

Ward, K. (2008, February 17 ). Breathing better is good business. The Associated Press State and Local Wire. Retrieved from http://www.lexisnexis.com

Whorton, J. C. (2002). Nature cures: The history of alternative medicine in America. Oxford, England: Oxford.

Yahoo!, Inc. (2011, May 19). Yahoo! reveals Oprah's effect on web searches. Business Wire. Retrieved from http://www.businesswire.com/news/home/20110519006704/en/Yahoo!-Reveals-Oprah'sEffect-Web-Searches

Zoller, H. M., \& Kline, K. N. (2008). Theoretical contributions of interpretive and critical research in health communication. In C. S. Beck (Ed.), Communication Yearbook, 32 (pp. 89-135). New York: Routledge. 


\section{Endnotes}

${ }^{1}$ James Reston was a US news reporter covering the Richard Nixon visit to China. He had an emergency appendectomy in China using only acupuncture for anesthesia.

${ }^{2}$ Before its mainstreaming, the neti pot was often affiliated with yoga practice (Frawley, 2005).

${ }^{3}$ Many scholars debate the correct terminology to use for this body of health therapies (see Baer, 2004).

In this article, we will use CIM (Sharf et al., 2013) as an overarching term that recognizes both that the alternative medicines of yesterday may be the integrated or complementary medicines of today and that what unites the treatments may be a larger holistic philosophy of health focused on the individual. 